\title{
Discovery of a dense molecular cloud towards a young massive embedded star in 30 Doradus *
}

\author{
M. Rubio ${ }^{1}$, S. Paron ${ }^{2}$, and G. Dubner ${ }^{2}$ \\ 1 Departamento de Astronomía, Universidad de Chile, Casilla 36-D, Santiago, Chile \\ e-mail: mrubio@das.uchile.cl \\ 2 Instituto de Astronomía y Física del Espacio (IAFE), CC 67, Suc. 28, 1428 Buenos Aires, Argentina \\ e-mail: sparon@iafe.uba.ar
}

Received 20 June 2008 / Accepted 29 June 2009

\section{ABSTRACT}

\begin{abstract}
Context. The 30 Doradus region in the Large Magellanic Cloud is one of the most outstanding star-forming regions of the Local Group and a primary target for studying star formation in an environment of low metallicity.

Aims. To obtain a more complete picture of the cool gas that has not yet been consumed or dispersed, we searched for line emission from molecular clouds that could be associated with molecular hydrogen emission detected in the region.

Methods. We obtained a high sensitivity ${ }^{12} \mathrm{CO} J=2-1$ map with the $15-\mathrm{m}$ SEST telescope, complemented by pointed observations of ${ }^{13} \mathrm{CO} J=2-1$ and $\mathrm{CS} J=2-1$.

Results. We report the discovery of a dense molecular cloud towards an embedded young massive star at $\sim 20^{\prime \prime}$ ( $\sim 5 \mathrm{pc}$, at the distance of $50 \mathrm{kpc}$ ) northwest of R136, the compact massive central stellar cluster powering 30 Doradus in the LMC, which could be triggering star formation in the surrounding molecular clouds. We derived a molecular mass of $\lesssim 10^{4} M_{\odot}$, a linear radius of $3 \mathrm{pc}$, as an upper limit, and a mean density of $\gtrsim 10^{3} \mathrm{~cm}^{-3}$ for the cloud. The detection of the CS $J=2-1$ emission line indicates higher densities, $\sim 10^{6} \mathrm{~cm}^{-3}$. The dense molecular cloud is associated with molecular $2.12 \mu \mathrm{m} \mathrm{H}_{2}$ emission. We suggest that the observed molecular gas could be the remains of dense molecular material surviving the action of strong UV fields and winds in which the young massive star has formed.
\end{abstract}

Key words. Magellanic Clouds - HII regions - ISM: individual objects: 30 Doradus - ISM: molecules

\section{Introduction}

In the Large Magellanic Cloud (LMC), 30 Doradus is the brightest giant star-forming region among all galaxies in the Local Group. It is 10 times more luminous than NGC3603, considered to be the most spectacular HII region in our Galaxy powered by a central compact massive cluster containing over $2000 M_{\odot}$

The 30 Doradus nebula is powered by a dense central compact cluster, R136. This cluster was initially thought to be a supermassive star $\left(1000 M_{\odot}\right)$ to explain the measured UV photons required to ionize the HII region. Several very massive stars in R136 and other hot stars around it (Walborn \& Blades 1997) emit a very large flux of Lyman continuum photons that ionize most of the surrounding gas, forming a super giant HII region. The stellar winds and possibly supernova explosions have eroded an asymmetric cavity, bounded on one side (the NW half) by denser material, seen in particular as molecular clouds (Johansson et al. 1998). Today we know that the cluster R136 consists of a compact dense core $(0.1 \mathrm{pc}$ in diameter), where more than $65 \mathrm{O} 3-$ type stars, the most luminous stars known, and more than $10 \mathrm{WR}$ stars have been disentangled with HST observations (Massey \& Hunter 1998). R136 has a stellar mass of $10^{5} M_{\odot}$ and a total luminosity of $\sim 10^{8} L_{\odot}$. The R136 cluster, the prototypical super star cluster (SSC), is visible by naked eye at a distance of $50 \mathrm{kpc}$.

* The observations were made with the Swedish-ESO Submillimeter Telescope, SEST, which was operated jointly by the Swedish National Facility for Radioastronomy and ESO.
In this sense, 30 Doradus is exceptional and the study of star formation and the molecular ambient in this HII region is crucial for understanding the star-forming processes around massive, compact SSCs interacting with low metallicity molecular clouds, which are exposed to high UV radiation fields and strong winds from WR and luminous $\mathrm{O}$ stars. The physical conditions in this ISM resemble those that existed in the early universe and thus can shed important light on the primeval process of star formation.

The 30 Doradus region has been extensively studied in optical and near infrared (NIR) wavelengths (Poglitsch et al. 1995; Rubio et al. 1998). Several bright embedded infrared sources (Rubio et al. 1998), an $\mathrm{H}_{2} \mathrm{O}$ maser and numerous nebular microstructures as revealed by Hubble Space Telescope (HST/WFPC2) images show active, as well as recent and ongoing, star formation (Walborn et al. 1999 and references therein).

In particular, Rubio et al. (1992) (RRG) reported for the first time an isolated IR source (source W9) located $20^{\prime \prime}$ northwest of R136. Based on the source position in the $J-H$ vs $H$ $K$ color-color diagram and its spectral energy distribution, the authors propose that W9 was most probably a young massive star. Ground-based infrared observations with high sensitivity and improved spatial resolution allowed for the discovery of several new bright embedded IR sources associated with molecular clouds in this region (Rubio et al. 1998). All of the RRG sources were identified, and some of them were resolved into several components. RRG-W9 was resolved into two sources, IRSW127 , a very bright object with the largest IR excesses among 


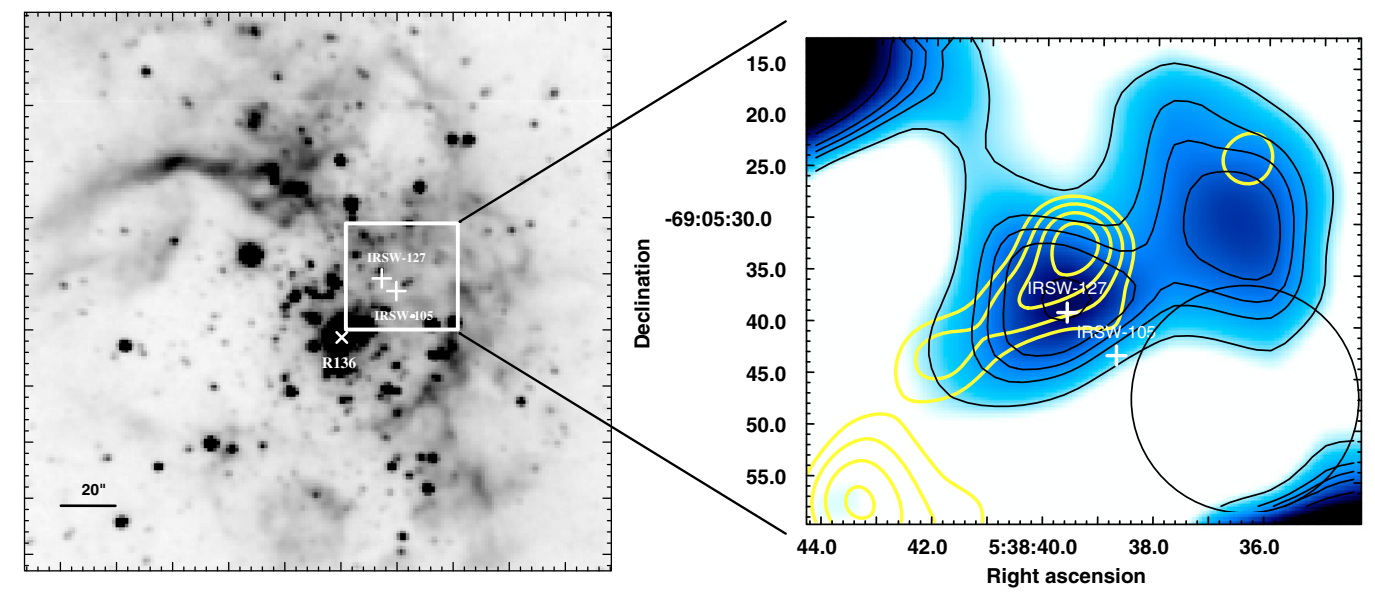

Fig. 1. Left: NIR $2.14 \mu \mathrm{m}$ continuum image of 30 Doradus showing the position of IRSW-105, IRSW-107 (crosses), and the center of R136 (the X). The box shows the extent of the map displayed in the right panel. Right: ${ }^{12} \mathrm{CO} J=2-1$ emission integrated between 232 and $254 \mathrm{~km} \mathrm{~s}{ }^{-1}$ towards the mentioned IR sources. The black contour levels are 5,6,6.5, 7, and $8 \mathrm{~K} \mathrm{~km} \mathrm{~s}^{-1}$. The yellow contours correspond to the $\mathrm{H}_{2}$ near infrared emission. The black circle is the ${ }^{12} \mathrm{CO} J=2-124^{\prime \prime}$ beam.

those in 30 Doradus $\left(K_{\mathrm{S}}=13.48, K_{\mathrm{S}}-H=2.85\right)$ and IRSW105 , displaced by $\sim 6^{\prime \prime}$. These two IR sources show spatial coincidence (within $\sim 10^{\prime \prime}$ ) with the O-type stars P621 and P600 classified as O3-O6 V by Walborn \& Blades (1997), who point out that they might be very young objects on or near the zero-age main sequence. As Rubio et al. (1998) suggest, the IR sources and the mentioned O-type stars likely represent the same starformation events.

Star formation occurs in molecular clouds. These clouds are expected to be destroyed by the violent interaction of the newly born massive stars. The two IR sources were found at only $0.5 \mathrm{pc}$ from the energetic massive cluster R136, and their proximity motivated us to study the molecular gas near the massive and luminous stellar cluster R136.

In this work, we present ${ }^{12} \mathrm{CO} J=2-1,{ }^{13} \mathrm{CO} J=2-1, \mathrm{CS}$ $J=2-1$, and ${ }^{12} \mathrm{CO} J=1-0$ data towards IRSW-105 and IRSW127.

\section{Observations}

High-sensitivity observations in ${ }^{12} \mathrm{CO} J=2-1$ (rest frequency: $230.538 \mathrm{GHz}$ ) were made between January and July, 1998 using the $15 \mathrm{~m}$ Swedish-ESO Submillimeter Telescope (SEST). The observations were done in position switch mode with a fixed reference off position free of $\mathrm{CO}$ emission. A narrowband AOS high-resolution (HRS) spectrometer with 2000 channels, $80 \mathrm{MHz}$ bandwidth, and $41.7 \mathrm{kHz}$ channel separation (corresponding to $0.054 \mathrm{~km} \mathrm{~s}^{-1}$ for the ${ }^{12} \mathrm{CO} J=2-1$ line) was used as back end. At $230 \mathrm{GHz}$ the beamwidth and main bean efficiency of the telescope were $24^{\prime \prime}$ and 0.60 , respectively. Calibration was done using the standard chopper wheel technique. The pointing accuracy, checked during the observations on RDor, was better than $2^{\prime \prime}$. Typical system temperatures were $\sim 200 \mathrm{~K}$. The observations had an rms noise of $0.07 \mathrm{~K}$, achieved after $4 \mathrm{~min}$ of integration in each position, which notably improved the sensitivity reached by Johansson et al. (1998) and Ott et al. (2008) in their ${ }^{12} \mathrm{CO}$ $J=1-0$ observations. Mapping was done with $10^{\prime \prime}$ spacings to produce fully sampled maps. The data were reduced using $\mathrm{CLASS}^{1}$, and linear and, in a few cases, third-order polynomia,

${ }^{1}$ CLASS is a GILDAS software for reduction and analysis of (sub)millimeter spectroscopic data. GILDAS, the "Grenoble Image and Line were used for baseline fitting. The spectra were smoothed to a velocity resolution of $0.25 \mathrm{~km} \mathrm{~s}^{-1}$.

The observations covered the region between the two strongest CO molecular clouds mapped in 30 Doradus (Cloud 10 and Cloud 6, Johansson et al. 1998) where no CO emission was detected by Johansson et al. (1998). However, Rubio et al. (1998) observed concentrations of vibrationally excited $\mathrm{H}_{2}$ at $2.12 \mu \mathrm{m}$ in this region, clearly calling for more sensitive $\mathrm{CO}$ observations. In this study, we restrict ourselves to the observations covering the area near the central cluster R136. This region is shown in Fig. 1 (left) with a $2.14 \mu \mathrm{m}$ continuum image of the 30 Doradus nebula. Two crosses show the position of the IR sources and an $X$ indicates the center position of R136. The box indicates the region that is analyzed in the ${ }^{12} \mathrm{CO} J=2-1$ line.

We also obtained ${ }^{13} \mathrm{CO}$ and $\mathrm{CS} J=2-1$ spectra towards the position of the strongest ${ }^{12} \mathrm{CO} J=2-1$ emission in the area, $\mathrm{RA}=5^{\mathrm{h}} 38^{\mathrm{m}} 39.5^{\mathrm{s}}, \mathrm{dec}=-69^{\circ} 05^{\prime} 37.5^{\prime \prime}(\mathrm{J} 2000)$. The telescope beam at the CS $J=2-1$ transition is $45^{\prime \prime}$ with a beam efficiency of 0.70 . These spectra were reduced in a similar way to the ${ }^{12} \mathrm{CO}$ $J=2-1$ observations. A ${ }^{12} \mathrm{CO} J=1-0$ spectrum towards this position was obtained from Johansson et al. (1998).

\section{Results and discussion}

Figure 1 (right) displays the velocity-integrated ${ }^{12} \mathrm{CO} J=2-1$ emission between 232 and $254 \mathrm{~km} \mathrm{~s}^{-1}$ covering an area of approximately $50^{\prime \prime} \times 50^{\prime \prime}$ around IRSW-127 (W9) and IRSW-105. The ${ }^{12} \mathrm{CO} J=2-1$ map probably shows two CO clumps. The strongest one has its maximum very near IRSW-127, and IRSW105 lies on its SW border. The second CO clump is seen towards the northwest and does not show any IR sources.

Previous studies of the ISM in 30 Doradus have shown that $\mathrm{H}_{2}$ structures appear intimately associated with bright IR sources, embedded $\mathrm{O}$ stars, and dense molecular clumps exposed to strong stellar radiation field (Poglitsch et al. 1995; Rubio et al. 1998). In Fig. 1 (right), we have included in yellow contours the $2.12 \mu \mathrm{m}$ molecular hydrogen emission extracted from Rubio et al. (1998). The $\mathrm{H}_{2}$ emission and the $\mathrm{CO}$ contours show a similar spatial distribution, with the IR source IRSW-127 associated with the brightest $\mathrm{H}_{2}$ and $\mathrm{CO}$ emission.

Data Analysis Software", is a collection of software developed by the Observatoire de Grenoble and IRAM. 
Table 1. ${ }^{12} \mathrm{CO} J=2-1$ molecular parameters of the strongest $\mathrm{CO}$ clump displayed in Fig. 1 (right).

\begin{tabular}{cccccc}
\hline \hline$T_{\mathrm{mb}}$ & $V_{\mathrm{c}}$ & $\Delta v$ & $I_{\mathrm{CO}}$ & $R$ & $L_{\mathrm{CO}}$ \\
\hline $0.65 \pm 0.05$ & $244.40 \pm 0.40$ & $4.40 \pm 0.50$ & $4.00 \pm 0.20$ & 3 & 120 \\
\hline
\end{tabular}

Table 1 presents the molecular parameters of the strongest CO clump displayed in Fig. 1 (right), which can also be appreciated in the $244 \mathrm{~km} \mathrm{~s}^{-1}$ velocity channel map in Fig. 2. $T_{\mathrm{mb}}$ is the peak brightness temperature in $\mathrm{K}, V_{\mathrm{c}}$ the central velocity in $\mathrm{km} \mathrm{s}^{-1}, \Delta v$ the line width (FWHM) in $\mathrm{km} \mathrm{s}^{-1}$, and $I$ the integrated line intensity in $\mathrm{K} \mathrm{km} \mathrm{s}^{-1}$. Errors are formal $1 \sigma$ value for the model of the Gaussian line shape.

To determine the size of the molecular clump we applied $R=$ $\left(\frac{N}{\pi}\right)^{1 / 2} \Delta s$, where $\Delta s$ is the linear grid spacing and $N$ the number of positions at which the cloud is detected above $\sim 5 \sigma_{\text {rms }}$. We obtain a radius of $\sim 3 \mathrm{pc}$. Since the angular size of the considered structure is comparable to the angular resolution of the present observations, a linear radius of 3 pc can be derived as an upper limit for the size of this clump. We derived the CO luminosity $L_{\mathrm{CO}}=120 \mathrm{~K} \mathrm{~km} \mathrm{~s}^{-1} \mathrm{pc}^{2}$, using $L_{\mathrm{CO}}=I_{\mathrm{CO}} N(\Delta s)^{2}$, where $I_{\mathrm{CO}}=$ $\int T_{\mathrm{mb}} \mathrm{d} v$. The radius (in pc) and the luminosity (in $\mathrm{K} \mathrm{km} \mathrm{s}^{-1} \mathrm{pc}^{2}$ ) are presented in the last two columns of Table 1.

In Fig. 2 we present channel maps of the velocity-integrated ${ }^{12} \mathrm{CO} J=2-1$ emission in steps of $\sim 2 \mathrm{~km} \mathrm{~s}^{-1}$ towards IRSW127 and IRSW-105. The two IR sources are located at the position of the peak and the SW border of ${ }^{12} \mathrm{CO} J=2-1$ emission in the $\sim 244 \mathrm{~km} \mathrm{~s}^{-1}$ velocity channel map. The spatial coincidence of IRSW-127 with the molecular clump peak and the large IR excess of this source, strongly suggests that IRSW-127 is embedded in this molecular clump. The $\mathrm{CO}$ emission is also seen at lower and higher velocity components in a different spatial location than that of the $\sim 244 \mathrm{~km} \mathrm{~s}^{-1}$ component. At lower velocities, the $\mathrm{CO}$ emission corresponds to an elongated structure extending from southeast to the center of the region. At higher velocities, the $\mathrm{CO}$ emission is associated with other molecular structure that contains the northwestern clump and others clumps farther away. This is evidence of a high degree of clumping in the region.

\subsection{Physical properties}

Figure 3 shows the spectra of the ${ }^{12} \mathrm{CO} J=1-0$ and $J=2-$ $1,{ }^{13} \mathrm{CO}$ and $\mathrm{CS} J=2-1$ transitions obtained towards the position of the strongest ${ }^{12} \mathrm{CO} J=2-1$ emission; RA = $5^{\mathrm{h}} 38^{\mathrm{m}} 39.5^{\mathrm{s}}$, Dec $=-69^{\circ} 05^{\prime} 37.5^{\prime \prime}(\mathrm{J} 2000)$. The spectra were Hanning-smoothed and are presented as histograms for a better display. In all the $\mathrm{CO}$ transitions, three velocity components are seen at $237 \mathrm{~km} \mathrm{~s}^{-1}, 244 \mathrm{~km} \mathrm{~s}^{-1}$, and $249 \mathrm{~km} \mathrm{~s}^{-1}$, respectively, while the CS $J=2-1$ spectrum only shows emission at the two higher velocity components. Table 2 summarizes the line emission parameters as obtained from Gaussian fit to each spectra.

We determined the line ratios of the strongest $\mathrm{CO}$ clump at the velocity component $\sim 244 \mathrm{~km} \mathrm{~s}^{-1}$. These ratios are presented in Table 3, which includes the intensity ratio and the velocityintegrated line-intensity ratio at the peak position convolved to the same beam size.

On the other hand, integrating the ${ }^{12} \mathrm{CO} J=1-0$ data from Johansson et al. (1998) around $\sim 244 \mathrm{~km} \mathrm{~s}^{-1}$, we found a weak emission at the position of our $\mathrm{CO}$ clump. Convolving the ${ }^{12} \mathrm{CO}$ $J=2-1$ map to the $J=1-0$ angular resolution, we obtained the ${ }^{12} \mathrm{CO} J=2-1$ and $J=1-0$ integrated line ratio $\left(R_{2-1 / 1-0}\right)$. For the CO clump, we obtained $R_{2-1 / 1-0} \sim 1.5$.
The value obtained in the isotopic line-integrated ratio agrees with those for molecular gas in other star forming-regions in the Magellanic Clouds (Israel et al. 2003) and compatible with a low metallicity and strong radiation field. The multiple-peak structure and the presence of wings in the spectra may indicate that the main contribution to the gas motion could be a turbulent velocity field (Falgarone et al. 1994; Garay et al. 2002).

The detection of CS $J=2-1$ towards this molecular clump implies the presence of dense gas. Generally, this "high-density" tracer indicates densities $\sim 10^{5} \mathrm{~cm}^{-3}$ with an upper limit of a few $\times 10^{6} \mathrm{~cm}^{-3}$ (Heikkilä et al. 1999; Wang et al. 2009). The CS $J=2-1$ line traces the dense interior of the clump, while the $\mathrm{CO}$ lines trace the external and less dense envelopes. The angular resolution of the CS $J=2-1$ observations is a factor of two lower than the ${ }^{12} \mathrm{CO} J=2-1$ observation, which implies that the CS spectra covers the total $50^{\prime \prime} \times 50^{\prime \prime}$ studied area. Thus the CS emission could be coming from several dense regions. The most intense CS component is seen at $\sim 249 \mathrm{~km} \mathrm{~s}^{-1}$, and a second weaker component is at $\sim 245 \mathrm{~km} \mathrm{~s}^{-1}$, very close to $\sim 244 \mathrm{~km} \mathrm{~s}^{-1}$ where the $\mathrm{CO}$ main component is seen. These different velocity components seen in the CS spectra could be more evidence of clumpiness of the region.

We determine the optical depth of the CO clump considering an isotopic abundance ratio $\left(\left[{ }^{12} \mathrm{CO} /{ }^{13} \mathrm{CO}\right]\right)$ between 40 and 50. Heikkilä et al. (1999) obtained $\left[{ }^{12} \mathrm{CO} /{ }^{13} \mathrm{CO}\right]$ ratios of 38 and $\left[\mathrm{HCO}^{+} / \mathrm{H}^{13} \mathrm{CO}^{+}\right]$ratios of $35 \pm 21$ in 30 Dor- 10 , and in the SMC N27 cloud, they obtained isotopic abundance ratios of 35 and 40-90, respectively. Recently, Wang et al. (2009) obtained the isotope ratio $\left[{ }^{12} \mathrm{C} /{ }^{13} \mathrm{C}\right] \sim 49 \pm 5$ in the star-forming region $\mathrm{N} 113$ in the LMC. Assuming $T_{\mathrm{ex}}=10 \mathrm{~K}$, that the surface filling factors and the excitation temperatures of the ${ }^{12} \mathrm{CO}$ and ${ }^{13} \mathrm{CO}$ emissions are equal, and using the ratio of the peak temperatures, we calculated the ${ }^{12} \mathrm{CO}$ and ${ }^{13} \mathrm{CO} J=2-1$ optical depths for this cloud. We obtained $\tau^{12} \sim 6$ and $\tau^{13} \sim 0.13$, respectively. This result would not be significantly altered if $T_{\mathrm{ex}}$ were as larger than $30 \mathrm{~K}$. The ${ }^{12} \mathrm{CO} J=2-1$ line appears optically thick, while the emission in the ${ }^{13} \mathrm{CO} J=2-1$ line is optically thin. The ratios obtained from our CO clump are similar to those obtained in N159 (Pineda et al. 2008). Different beam filling factors play a minor role in the line ratios, even in a clumpy medium (Gierens et al. 1992; Pineda et al. 2008).

\subsection{Molecular mass}

We used three independent ways to estimate the molecular mass of the discovered molecular clump. First, we assumed that the molecular clump is in virial equilibrium. Assuming that the clump has a spherical shape, a radius of $3 \mathrm{pc}$, which is an upper limit, we estimated $M_{\text {vir }} \lesssim 1 \times 10^{4} M_{\odot}$, using the constant $B=190$ for a density profile of $\rho(r) \propto 1 / r$ (MacLaren et al. 1988). Second, we estimated the molecular clump mass from the observed ${ }^{12} \mathrm{CO} J=2-1$ luminosity using an $\mathrm{H}_{2}-{ }^{12} \mathrm{CO}(1-0)$ conversion factor of $X \sim 7 \times 10^{20} \mathrm{~cm}^{-2} \mathrm{~K}^{-1} \mathrm{~km}^{-1} \mathrm{~s}, 2.5$ times the Galactic value, as determined for this LMC region (Garay et al. 2002; Israel et al. 2003 and reference therein). We multiplied this conversion factor by the integrated line ratio $R_{2-1 / 1-0}=1.5$, obtained for the studied CO clump obtaining a molecular mass of $M_{\mathrm{CO}} \lesssim 2.7 \times 10^{3} M_{\odot}$. Third, we derived the mass using the ${ }^{13} \mathrm{CO} J=2-1$ emission and assuming LTE conditions. We assumed optically thick ${ }^{12} \mathrm{CO} J=2-1$ emission and a beam filling factor of 1 , which may not be completely true but allows us to make a first initial guess. We derived an optical depth, $\tau^{13} \sim 0.12$ and used the fractional abundance of $\left[\mathrm{H}_{2} /{ }^{13} \mathrm{CO}\right]$ $\sim 1.5 \times 10^{7}$ for this region (Heikkilä et al. 1999) we obtained 


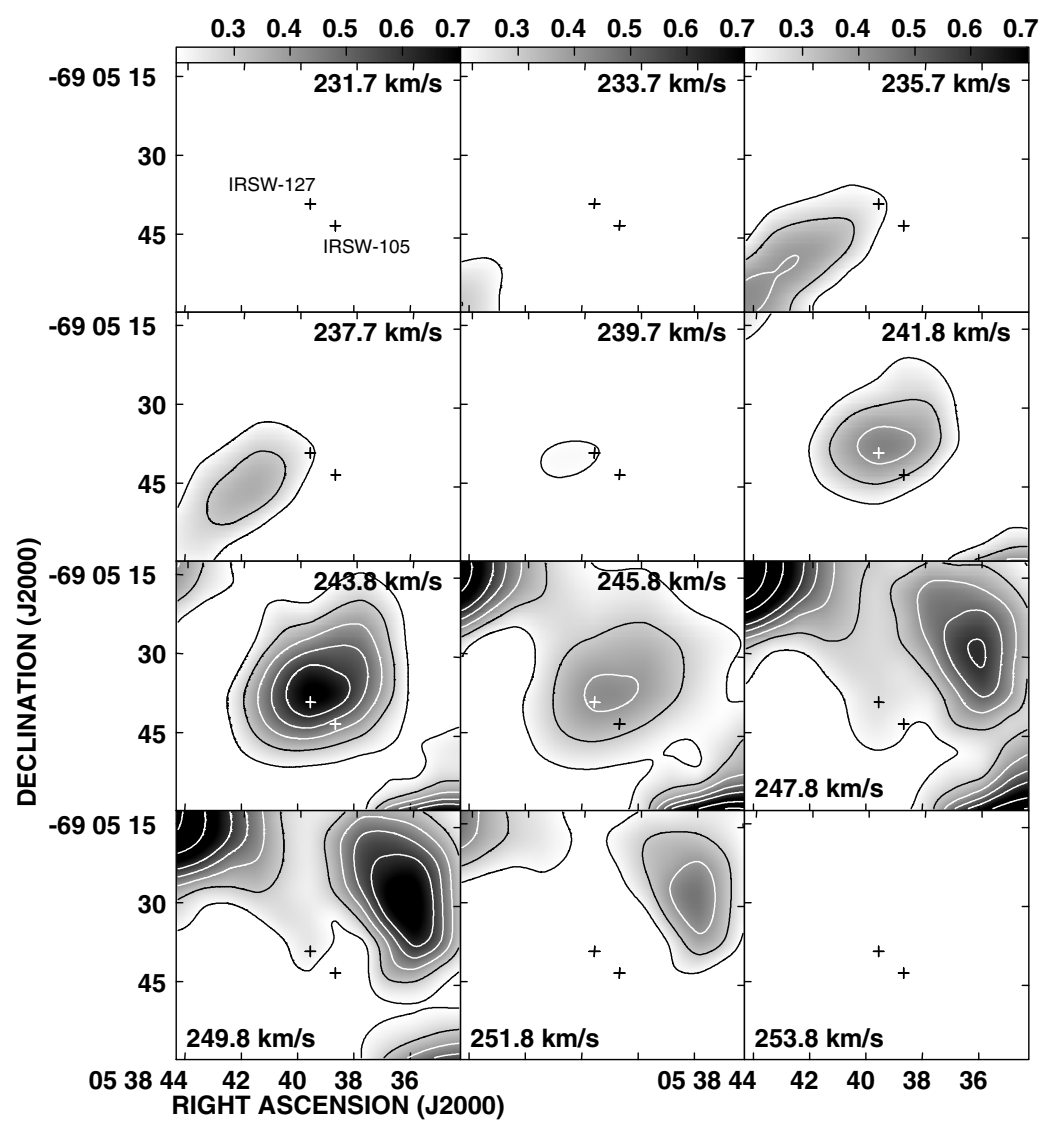

Fig. 2. ${ }^{12} \mathrm{CO} J=2-1$ emission integrated over velocity intervals of $\sim 2 \mathrm{~km} \mathrm{~s}^{-1}$. The contour levels are $0.2,0.3,0.4,0.5,0.6,0.8$, and $1 \mathrm{~K} \mathrm{~km} \mathrm{~s}{ }^{-1}$. The IR sources IRSW-105 and IRSW-127 are indicated by crosses.

Table 2. Observed parameters from the spectra at the peak position $\mathrm{RA}=5^{\mathrm{h}} 38^{\mathrm{m}} 39.5^{\mathrm{s}}, \mathrm{Dec}=-69^{\circ} 05^{\prime} 37.5^{\prime \prime}(\mathrm{J} 2000)$.

\begin{tabular}{ccccc}
\hline \hline Line emission & $\begin{array}{c}V_{\mathrm{c}} \\
\left(\mathrm{km} \mathrm{s}^{-1}\right)\end{array}$ & $\begin{array}{c}T_{\mathrm{mb}} \\
(\mathrm{K})\end{array}$ & $\begin{array}{c}\Delta v \\
\left(\mathrm{~km} \mathrm{~s}^{-1}\right)\end{array}$ & $\begin{array}{c}I \\
\left(\mathrm{~K} \mathrm{~km} \mathrm{~s}^{-1}\right)\end{array}$ \\
${ }^{12} \mathrm{CO} J=1-0$ & $237.6 \pm 0.9$ & $0.11 \pm 0.04$ & $2.1 \pm 0.2$ & $0.30 \pm 0.12$ \\
& $244.9 \pm 0.8$ & $0.50 \pm 0.06$ & $3.8 \pm 0.4$ & $1.82 \pm 0.25$ \\
& $251.4 \pm 0.9$ & $0.25 \pm 0.05$ & $5.6 \pm 0.2$ & $1.33 \pm 0.25$ \\
${ }^{12} \mathrm{CO} J=2-1$ & $237.8 \pm 0.9$ & $0.30 \pm 0.05$ & $5.7 \pm 0.5$ & $1.40 \pm 0.25$ \\
& $244.4 \pm 0.7$ & $1.00 \pm 0.08$ & $4.0 \pm 0.5$ & $5.20 \pm 0.25$ \\
& $249.7 \pm 0.8$ & $0.37 \pm 0.05$ & $5.5 \pm 0.7$ & $1.60 \pm 0.22$ \\
${ }^{13} \mathrm{CO} J=2-1$ & $236.7 \pm 0.3$ & $0.08 \pm 0.02$ & $0.4 \pm 0.3$ & $0.07 \pm 0.02$ \\
& $244.4 \pm 0.3$ & $0.13 \pm 0.01$ & $3.1 \pm 0.6$ & $0.40 \pm 0.04$ \\
$\mathrm{CS} J=2-1$ & $249.8 \pm 0.2$ & $0.07 \pm 0.01$ & $2.1 \pm 0.6$ & $0.16 \pm 0.03$ \\
& $245.7 \pm 0.4$ & $0.03 \pm 0.01$ & $1.7 \pm 0.6$ & $0.07 \pm 0.02$ \\
& $249.5 \pm 0.2$ & $0.07 \pm 0.01$ & $2.1 \pm 0.6$ & $0.12 \pm 0.03$ \\
\hline
\end{tabular}

Table 3. Line ratios for the $244 \mathrm{~km} \mathrm{~s}^{-1}$ component at the peak position.

\begin{tabular}{cccc}
\hline \hline Peak line ratio & $\frac{{ }^{12} \mathrm{CO}(2-1)}{{ }^{12} \mathrm{CO}(1-0)}$ & $\frac{{ }^{12} \mathrm{CO}(2-1)}{{ }^{13} \mathrm{CO}(2-1)}$ & $\frac{12 \mathrm{CO}(2-1)}{\mathrm{CS}(2-1)}$ \\
\hline Intensity: $T_{\mathrm{mb}}$ & $1 \pm 0.2$ & $8 \pm 1.2$ & $15 \pm 7$ \\
Vel. Integ.: $\int T_{\mathrm{mb}} \mathrm{d} v$ & $1.4 \pm 0.3$ & $13 \pm 2$ & $38 \pm 30$ \\
\hline
\end{tabular}

$M_{\text {LTE }} \lesssim 1.5 \times 10^{4} M_{\odot}$. As a result, we derived a number density of $\mathrm{H}_{2}$ molecules of $n_{\mathrm{vir}} \gtrsim 2.0 \times 10^{3} \mathrm{~cm}^{-3}, n_{\mathrm{CO}} \gtrsim 0.5 \times 10^{3} \mathrm{~cm}^{-3}$, and $n_{\mathrm{LTE}} \gtrsim 2.8 \times 10^{3} \mathrm{~cm}^{-3}$.

Considering that the mass values are upper limits, in what follows we perform a rough comparison between them. The virial mass and the LTE mass are similar, with the LTE mass slightly higher, implying that the molecular clump may be approximately in virial equilibrium. The mass obtained from the observed CO luminosity is approximately one order of magnitude lower than the virial and LTE masses. Such a difference may be indicative of the strong photo-dissociation that this molecular clump has suffered due to its location in extreme environmental conditions. The molecular clump may have a large $\mathrm{H}_{2}$ envelope where most of the mass is found with only a dense $\mathrm{CO}$ core which has survived from photo-dissociation (Lequeux et al. 1994). Another possibility for reconciling the masses estimated in different ways is by assuming a conversion factor 13 times larger than the Galactic value, as obtained by Garay et al. (1993) for molecular clouds in the 30 Doradus halo. Using this conversion factor, we obtain a molecular mass from the observed $\mathrm{CO}$ luminosity of $M_{\mathrm{CO}} \lesssim 1.4 \times 10^{4} M_{\odot}$, in agreement with the LTE mass.

\section{Conclusions}

We discovered a molecular clump located $220^{\prime \prime}$ northwest of the center of the compact cluster R136, where the IR source IRSW127 , a young massive star, is very likely embedded. This source appears very strongly correlated to the molecular maximum. We derived a clump radius of $3 \mathrm{pc}$ as an upper limit, a molecular mass of $\lesssim 10^{4} M_{\odot}$, and a density of $\gtrsim 10^{3} \mathrm{~cm}^{-3}$ from the ${ }^{12} \mathrm{CO} \mathrm{J}=$ $2-1$ emission. The $R_{2-1 / 1-0} \sim 1.5$, higher than the average value of $R_{2-1 / 1-0} \sim 0.8$ in a 9 arcmin beam, is consistent with optically 


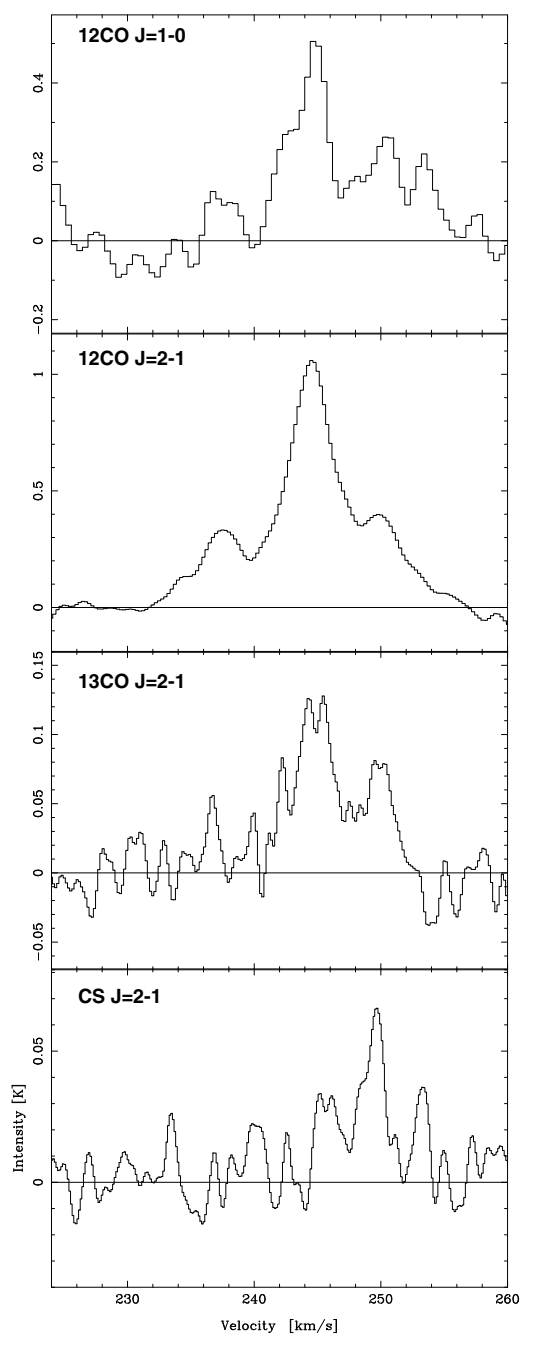

Fig. 3. ${ }^{12} \mathrm{CO}(1-0),{ }^{12} \mathrm{CO}(2-1),{ }^{13} \mathrm{CO}(2-1)$, and $\mathrm{CS}(2-1)$ spectra observed at RA $=5^{\mathrm{h}} 38^{\mathrm{m}} 39.5^{\mathrm{s}}$, dec $=-69^{\circ} 05^{\prime} 37.5^{\prime \prime}(\mathrm{J} 2000)$. The intensity scale that is in $T_{\mathrm{mb}}$ has been adjusted to better show the emission line.

thick, thermalized, dense gas $\left(\geq 10^{3} \mathrm{~cm}^{-3}\right)$ ready to form stars as pointed out by Sorai et al. (2001).

The molecular clump lies in a region of $\mathrm{H}_{2}$ emission. Poglitsch et al. (1995) suggest that the clumpy $\mathrm{H}_{2}$ emission that they observed in 30 Doradus may be coming from photodissociation regions (PDRs) and could be produced in dense $\sim 10^{6} \mathrm{~cm}^{-3}$ molecular clumps exposed to the stellar radiation field. The molecular clump studied in this work is exposed to an UV field of $\sim 6000$ times the average Galactic UV radiation field as determined using the Starburts99 code (Leitherer et al. 1999) for a 2 Myr cluster with a metallicity of 0.008 at the distance of $20^{\prime \prime}$ of R136 (Guzmán, private communication). Thus, the clump could have a large envelope of self-protected molecular gas.

Our detection of CS $J=2-1$ emission confirms the presence of dense gas, $\sim 10^{6} \mathrm{~cm}^{-3}$, in the molecular clump. It is detected at $\sim 245 \mathrm{~km} \mathrm{~s}^{-1}$, very close to the velocity component where the ${ }^{12} \mathrm{CO} \mathrm{J}=2-1$ emission is stronger and spatially correlated with the two IR sources. Although the CS $J=2-1$ emission is stronger at the $\sim 249 \mathrm{~km} \mathrm{~s}^{-1}$ velocity component, the $\mathrm{CO}$ emission at this velocity does not show any spatial coincidence with the IR sources. Thus, this CS emission could come from another dense clump where massive star formation has not yet occurred; or if it has occurred, the possible IR sources would not been detected by existing surveys because they could suffer an even greater extinction than that of IRSW127. Higher angular resolution observations of this high-density tracer would be needed to study the dense gas structure of the region.

As mentioned above, the CS velocity structure shows evidence of clumpiness, which agrees with previous results for the 30 Doradus nebula (Johansson et al. 1998; Heikkilä et al. 1999) and is compatible with star-forming activity. We find additional evidence of this activity: (1) the LTE mass $\left(M_{\mathrm{LTE}}\right)$ is slightly higher than the virial mass $\left(M_{\mathrm{vir}}\right)$, which according to Kawamura et al. (1998) and Tachihara et al. (2000) could be interpreted as an indication of star formation; and (2) the isotopic line integrated ratio $\left({ }^{12} \mathrm{CO} /{ }^{13} \mathrm{CO}\right)$ agrees with those found in different star-forming regions in the Magellanic Clouds.

We conclude that the discovered molecular clump could be the remains of molecular material that has survived the interaction of the strong winds and the UV field of R136. This CO clump is an example of a dense molecular cloud with a large fraction of its mass in $\mathrm{H}_{2}$ and not in $\mathrm{CO}$, as this molecule has suffered strong photo-dissociation from the UV radiation flux of the massive central cluster R136.

We confirm the suggestions that possible energetic winds and strong UV radiation from the stellar cluster R136 could be triggering the star formation in the surrounding molecular clouds and that IRSW-127 could be a newly formed young massive star embedded in a dense molecular clump.

Acknowledgements. M.R. is supported by the Chilean Center for Astrophysics FONDAP No. 15010003. S.P. and G.D. are members of the Carrera del investigador científico of CONICET, Argentina. We would like to thank Viviana Guzman for computing the UV radiation field from R136. This work was partially supported by the CONICET grant 6433/05, UBACYT A023 and ANPCYT PICT 04-14018. M.R. and S.P. acknowledge support from FONDECYT $\mathrm{N}^{\circ} 1080335$.

\section{References}

Falgarone, E., Lis, D. C., Phillips, T. G., et al. 1994, ApJ, 436, 728 Garay, G., Rubio, M., Ramirez, S., Johansson, L., \& Thaddeus, P. 1993, A\&A, 274,743

Garay, G., Johansson, L., Nyman, L.-Å., et al. 2002, A\&A, 389, 977 Gierens, K. M., Stutzki, J., \& Winnewisser, G. 1992, A\&A, 259, 271 Heikkilä, A., Johansson, L., \& Olofsson, H. 1999, A\&A, 344, 817

Israel, F. P., Johansson, L., Rubio, M., et al. 2003, A\&A, 406, 817 Johansson, L., Greve, A., Booth, R., et al. 1998, A\&A, 331, 857 Kawamura, A., Onishi, T., Yonekura, Y., et al. 1998, ApJS, 117, 387 Leitherer, C., Schaerer, D., Goldader, J. D., et al. 1999, ApJS, 123, 3 Lequeux, J., Le Bourlot, J., Des Forets, G. P., et al. 1994, A\&A, 292, 371 MacLaren, I., Richardson, K. M., \& Wolfendale, A. W. 1988, ApJ, 333, 821 Massey, P., \& Hunter, D. A. 1998, ApJ, 493, 180 Ott, J., Wong, T., Pineda, J. L., et al. 2008, Publ. Astron. Soc. Austr., 25, 129 Pineda, J. L., Mizuno, N., Stutzki, J., et al. 2008, A\&A, 482, 197 Poglitsch, A., Krabbe, A., Madden, S. C., et al. A. 1995, ApJ, 454, 293 Rubio, M., Roth, M., \& Garcia, J. 1992, A\&A, 261, L29

Rubio, M., Barbá, R. H., Walborn, N. R., et al. 1998, AJ, 116, 1708 Sorai, K., Hasegawa, T., Booth, R. S., et al. 2001, ApJ, 551, 794 Tachihara, K., Mizuno, A., \& Fukui, Y. 2000, ApJ, 528, 817 Walborn, N. R., \& Blades, J. C. 1997, ApJS, 112, 457

Walborn, N. R., Drissen, L., Parker, J. W., et al. 1999, AJ, 118, 1684 Wang, M., Chin, Y.-N., Henkel, C., Whiteoak, J. B., \& Cunningham, M. 2009, ApJ, 690, 580 